\title{
Cavitation Erosion Modelling on a Radial Divergent Test Section Using RANS
}

\author{
Luka Kevorkijan - Luka Lešnik - Ignacijo Biluš* \\ University of Maribor, Faculty of Mechanical Engineering, Slovenia
}

Cavitation is the phenomenon of fluid evaporation in hydraulic systems, which occurs due to a pressure drop below the value of the vapor pressure. For numerical modelling of this generally undesirable phenomenon, which is often associated with material damage (erosion), there are various mathematical vapor transfer models that have been validated in the past. There are different approaches to predicting cavitation erosion, which have mostly been experimental in the past. Recently various numerical models have been developed with the development of numerical simulations. They describe the phenomenon of cavitation erosion based on different theoretical considerations, such as Pressure wave hypothesis, Microjet hypothesis, or a combination of both.

In the present paper, an analysis of the Schnerr-Sauer transport cavitation model was used, upgraded with an erosive potential energy model based on pressure wave hypothesis for cavitation erosion prediction. The extended numerical model has been applied to the case of a radial divergent test section in three different mathematical formulations. The results of simulation were compared and validated to experimental work performed by other authors. The study shows that the distribution of surface accumulated energy agrees with the experimental results, although certain differences exist between formulations. The applied method appears to be appropriate for further use, and to be extended to materials response modelling in the future.

\section{Keywords: cavitation, erosion, erosive potential energy, numerical simulation}

\section{Highlights}

- The cavitation erosion process can be predicted by cavitation potential energy modelling.

- Numerical definition of erosive power is possible with different formulations of cavitation potential.

- Cavitation energy focusing accomplishes accurate prediction of cavitation erosion damage.

\section{INTRODUCTION}

Cavitation is the phenomenon that consists of the formation, activity and collapse of vapor structures inside a liquid medium. Usually, the phenomenon is undesired, since it causes many negative effects. Among those negative effects the cavitation erosion is the most complex one, since it combines complicated hydrodynamics with solid material response at the mechanical and metallurgical perspectives. The research of cavitation phenomenon dates to the late 19th and early 20th centuries. In 1917, Lord Rayleigh [1] derived the equation for calculation of the pressure in a liquid during the collapse of a spherical bubble. In 1949, Plesset [2] upgraded this work by writing the differential equation of the dynamics of a spherical bubble, today known as the Rayleigh-Plesset equation. Mostly experimental research of cavitation damage was conducted during the theoretical research of the cavitation phenomenon.. Hammit [3] studied cavitation damage on the case of fluid flow through a Venturi channel, and introduced the erosion model based on the energy spectrum of a vapor cavity. Vogel et al. [4] observed the dynamics of the collapse of a bubble along a solid wall, and derived the equation for calculation of the potential energy of the collapse.
By the end of the 20th century, two hypotheses were known about the origin of cavitation damage. The first hypothesis followed the high value of the pressure in the liquid around the bubble as it was calculated by Lord Rayleigh [1]. The existence of a pressure wave was confirmed by many experimental studies, for example, by Harrison [5]. A second, "microjet" hypothesis was proposed by Kornfeld and Suvorov [6], while Plesset and Chapman [7] predicted the microjet velocity numerically, and claimed that a microjet can damage the material.

Based on the pressure wave hypothesis, Fortes Patella et al. [8] presented an approach to erosion assessment with an energy cascade that transfers the initial potential energy of large vapor structures to the smallest structures, and eventually to the surface walls. A key contribution was the definition of the potential power of cavitation structures based on the total derivative of potential energy. The model upgrade is described in a paper of Leclercq et al. [9], where all of the vapor structures, even those not in direct contact with the wall, were considered. In the paper by Carrat et al. [10] the results of numerical analysis, including the amplitude and frequency of the pressure waves, were presented and validated experimentally. Based on their findings, Schenke and Terwisga [11] proposed 
a model of forecasting the erosion threat where energy generated by the vapor structures is conserved and transferred to the wall surface via the pressure wave released after the collapse. They also introduced a continuous formulation of energy transfer from wall-distant structures to the wall surface. Li [12] introduced the erosion intensity function, which is based on pressure only.

On the other hand, Dular et al. presented the semi-empirical cavitation erosion model based on the microjet hypothesis [13]. The same research group have contributed a lot mainly to the experimental field of cavitation erosion research, [14] and [15]. Based on their experimental findings and semi-empirical model, Peters et al. [16] introduced the multilevel modelling of two-phase flow, where the Euler-Euler and EulerLagrange approach were combined.

Currently, the research contributions are focused on the use of existing models on practical problem geometries [17], which are, in fact, more complex than in the past, due mainly to the rapid progress of computational capabilities [18]. Brunhart et al. [19] introduced the use of erosion indicators on the case of gaps within a diesel fuel pump. Gomez Santos et al. [20], similarly, presented the comparison of erosion models in engine injectors operating with ethanol. Despite intensive research work and numerous publications in the field of cavitation erosion, the derived models have still not been perfected, and offer many opportunities for upgrading and improvement. The reason for this is mainly the lack of consensus on the predominant mechanism of cavitation erosion. Following this, Arabnejad et al. [21] joined both erosion mechanisms. In their work, the collapse induced kinetic energy is divided into the kinetic energy of the pressure wave and kinetic energy of the microjet.

In the present work, a model by Schenke and Terwisga [11] has been applied to a radial divergent test section, which is a common test case for cavitation studies found in the literature. The mesh was generated with a meshing module within ANSYS Fluent 2020 R2, cavitating flow and cavitation erosion were calculated using the computational fluid dynamics (CFD) software ANSYS Fluent 2020 $\mathrm{R} 2$, where the model to predict cavitation erosion by Schenke and Terwisga [11] was implemented via a user defined function (UDF) which was written in the $\mathrm{C}$ programming language, compiled and linked to the solver.

Different authors analyzed cavitation on this flow case numerically, but they applied different cavitation erosion models [16]. In this work, we applied an erosive potential energy model based on the pressure wave hypothesis with three different mathematical formulations. The transfer of energy from the cavitation structures to the surface was accomplished with and without the energy focusing method. The numerical results show good trends and agreement with the experimental results, with certain limitations, which could be a subject of further studies and model optimizations.

\section{CAVITATION EROSION}

\subsection{Homogeneous Mixture Method}

In the following, we focus on the general formulation, and treat the fluid as a continuous mixture consisting of the volume fraction of the vapor phase and the volume fraction of the liquid phase $(1-\alpha)$. The flow is considered as isothermal, pure phases are treated as incompressible. The mixing rule applies to the properties of the mixture, the mixture density $(\rho)$ and the mixture viscosity $(\mu)$

$$
\begin{aligned}
& \rho=\alpha \rho_{v}+(1-\alpha) \rho_{l}, \\
& \mu=\alpha \mu_{v}+(1-\alpha) \mu_{l},
\end{aligned}
$$

where the liquid and vapor densities are $\rho_{l}=$ $998.85 \mathrm{~kg} / \mathrm{m}^{3}$ and $\rho_{v}=0.01389 \mathrm{~kg} / \mathrm{m}^{3}$. The liquid and vapor viscosities are $\mu_{l}=0.0011 \mathrm{~Pa} \cdot \mathrm{s}$ and $\mu_{v}=9.63$. $10^{-6} \mathrm{~Pa} \cdot \mathrm{s}$.

\subsection{Conservation of Vapor Phase Mass}

The vapor mass is defined as $m_{v}=\rho_{v} V_{v}=\rho_{v} \alpha V$, where $V_{v}$ is the vapor volume and $V$ is the mixture volume. According to this, the mass flow of vapor is

$$
\dot{m}_{v}=\int_{V} \frac{D m_{v}}{D t} d V=\int_{V} \frac{D \rho_{v} \alpha V}{D t} d V,
$$

and the source of the vapor fraction is

$$
\int_{V}\left[\frac{\partial \rho_{\nu} \alpha}{\partial t}+\vec{\nabla} \cdot\left(\rho_{v} \vec{u} \alpha\right)\right] d V=\int_{V} \rho_{v} S_{\alpha \nu} d V,
$$

where $\vec{u}$ is mixture velocity. If we write Eq. (4) in differential form and consider pure phases incompressible, we get

$$
\frac{\partial \alpha}{\partial t}+\vec{\nabla} \cdot(\alpha \vec{u})=S_{\alpha \nu},
$$

where $S_{\alpha \nu}$ presents the source of the vapor phase. 


\subsection{Conservation of the Mixture Mass}

Similarly to Eq. (5), the mass conservation for the mixture is written as

$$
\frac{\partial \rho}{\partial t}+\vec{\nabla} \cdot(\rho \vec{u})=0
$$

From $\rho=\alpha \rho_{v}+(1-\alpha) \rho_{l}$ and $\alpha=\frac{\rho-\rho_{l}}{\rho_{v}-\rho_{l}}$ we
can write

$$
\frac{\partial \alpha}{\partial t}+\vec{u} \cdot \vec{\nabla} \alpha=-\alpha \vec{\nabla} \cdot \vec{u}-\frac{\rho_{l}}{\left(\rho_{v}-\rho_{l}\right)} \vec{\nabla} \cdot \vec{u},
$$

and after rearrangement

$$
\frac{\partial \alpha}{\partial t}+\vec{u} \cdot \vec{\nabla} \alpha=-\frac{\rho}{\rho_{v}-\rho_{l}} \vec{\nabla} \cdot \vec{u},
$$

which presents the conservation equation of the mixture mass written by the vapor volume fraction $\alpha$.

\subsection{Conservation of the Mixture Momentum}

Within a homogeneous mixture, the approach for the momentum conservation is written for the mixture as

$$
\frac{\partial(\rho \vec{u})}{\partial t}+\vec{\nabla} \cdot(\rho \vec{u} \vec{u})=-\vec{\nabla} p+\vec{\nabla} \cdot \tau,
$$

where $\tau$ is the viscous stress tensor and is evaluated as

$$
\tau=\mu\left(\vec{\nabla} \vec{u}+\vec{\nabla} \vec{u}^{T}\right)
$$

From Eq. (9) it is noticeable that body forces (gravity) are omitted in this work. The flow was considered as incompressible, while compressible behavior of the mixture is exhibited during the phase transition, as in the work of [21] and [22].

\subsection{Turbulence Modelling}

To model turbulent flow, the RANS approach was adopted, with the SST $k-\omega$ model. In order to address the transient cavitation effects that arise due to the compressibility of a liquid-vapor mixture, the turbulent viscosity $\mu_{t}$ is modified according to Reboud et al. [23], and Coutier-Delgosha et al. [24] as follows

$$
\mu_{t}=\frac{f(\rho) k}{\omega} \frac{1}{\max \left[\frac{1}{\alpha^{*}}, \frac{S F_{2}}{a_{1} \omega}\right]},
$$

where $k$ is the turbulence kinetic energy, $\omega$ is the specific rate of dissipation of the turbulence kinetic energy, $\alpha^{*}$ is a damping coefficient, $S$ is the strain rate magnitude, $F_{2}$ is the second blending function and $\alpha_{1}$ is the model constant equaling 0.31 . Correction of turbulent viscosity is achieved by modifying the mixture density function $f(\rho)$ such that a lower turbulent viscosity is achieved in the presence of both the liquid and vapor phases

$$
f(\rho)=\rho_{v}+\frac{\left(\rho-\rho_{v}\right)^{n}}{\left(\rho_{l}-\rho_{v}\right)^{n-1}},
$$

where $n$ is an arbitrary exponent with a recommended value of 10 .

This modification of turbulent viscosity was implemented in ANSYS Fluent via a UDF.

\subsection{Cavitation Modelling}

For the cavitation modelling, the additional conservation equation of vapor mass is solved.

$$
\frac{\partial \alpha_{v}}{\partial t}+\vec{\nabla} \cdot\left(\vec{u}_{i} \alpha_{v}\right)=\mathrm{S}_{\alpha_{v}} .
$$

The Schnerr-Sauer cavitation model was used, which states that mass transfer source term $\mathrm{S}_{\alpha_{v}}$ is

$$
\mathrm{S}_{\alpha_{v}}=\left\{\begin{array}{l}
\frac{\rho_{v} \rho_{l}}{\rho} \alpha_{v}\left(1-\alpha_{v}\right) \frac{3}{R_{b}} \sqrt{\frac{2}{3} \frac{\left(p_{v}-p\right)}{\rho_{l}}} \text { if } p<p_{v} \\
\frac{\rho_{v} \rho_{l}}{\rho} \alpha_{v}\left(1-\alpha_{v}\right) \frac{3}{R_{b}} \sqrt{\frac{2}{3} \frac{\left(p-p_{v}\right)}{\rho_{l}}} \text { if } p>p_{v}
\end{array},\right.
$$

where the bubble radius is

$$
R_{b}=\left(\frac{\alpha_{v}}{n_{0} \frac{4}{3} \pi\left(1-\alpha_{v}\right)}\right)^{1 / 3} .
$$

The bubble number density was specified with a value $1 \cdot 10^{11}$. The vapor pressure was set to $p_{v}=1854$ $\mathrm{Pa}$.

\subsection{Cavitation Erosion Potential}

Hammit [3] and Vogel et al. [4] described the potential energy of a cavitation bubble in terms of work done as a consequence of the pressure difference between the vapor inside the bubble and the surrounding liquid. If we analyze the spherical cavitation bubble with volume $V_{V}$, then the potential energy of the cavitating bubble $E_{\text {pot }}$ equals

$$
E_{p o t}=\left(p_{d}-p_{v}\right) V_{V},
$$

where $p_{d}$ is the pressure in the surrounding liquid and $p_{v}$ is the vapor pressure inside the bubble. Authors 
[11] highlighted the problem of defining $p_{d}$ for the purposes of numerical simulations, where the pressure in the surrounding liquid is evaluated locally in each computational grid cell. In that case $p_{d}$ equals $p_{v}$ and the calculated potential energy equals zero. In this work $p_{d}$ is taken as a time-averaged value in each computational grid cell as proposed in [11]

$$
p_{d}=\frac{1}{t^{*}} \int_{0}^{t^{*}} p(t) d t
$$

where $p(t)$ is an instantaneous pressure that is then time-averaged over the current simulated time $t^{*}$.

The cavitation potential power $P_{p o t}$ is introduced as the total derivative of the potential energy $E_{p o t}$

$$
P_{p o t}=\frac{D\left(p_{d}-p_{v}\right)}{D t} V_{V}+\frac{D V_{V}}{D t}\left(p_{d}-p_{v}\right)
$$

Since $p_{v}=$ const. (isothermal flow) and $p_{d}=f(\vec{x}, t) \neq$ const. the potential power equals

$$
P_{p o t}=\frac{D p_{d}}{D t} V_{V}+\frac{D V_{V}}{D t}\left(p_{d}-p_{v}\right) .
$$

The first term in Eq. (19) is neglected, as it has been found that it is at least an order of magnitude lower than the second term [22]. Finally, the cavitation potential power is written as

$$
P_{p o t}=\frac{D V_{V}}{D t}\left(p_{d}-p_{v}\right) .
$$

To calculate the cavitation potential power numerically, it is appropriate to calculate the instantaneous change of the volume specific potential energy [11] or cavitation erosion potential

$$
\dot{e}_{p o t}=\frac{P_{p o t}}{V_{c e l l}},
$$

where $V_{\text {cell }}$ presents the volume of the computational grid cell. A positive value of $\dot{e}_{p o t}$ is then a consequence of an increase of the vapor cavity volume, and a negative value is a consequence of the reduction in cavity volume. It is possible to rearrange Eq. (20) and rewrite it in modified forms, dependent on different variables. In the present study we used three equation forms, namely, a form dependent on the vapor volume fraction $e_{p o t, \alpha}$, a form dependent on the velocity divergence $e_{p o t, \mathrm{div} u}$ and a form dependent on the vapor phase source term $e_{p o t, S_{\alpha v}}$.

\subsubsection{Cavitation Erosion Potential $\dot{e}_{p o t, \alpha}$}

If we calculate the total derivative in Eq. (16), consider the relations $V \rightarrow V_{\text {cell }}$ and $V_{\text {cell }}=$ const. and combine it with Eq. (21) we get

$$
\dot{e}_{p o t, \alpha}=\left(\frac{\partial \alpha}{\partial t}+\vec{u} \cdot \vec{\nabla} \alpha\right)\left(p_{d}-p_{v}\right)
$$

Eq. (22) defines the cavitation erosion potential with vapor volume fraction $\alpha$. It describes the change of potential due to the bubble volume decrease during collapse.

\subsubsection{Cavitation Erosion Potential $\dot{e}_{p o t, d i v u}$}

Combining the mass conservation Eq. (8) and $\alpha=\frac{\rho-\rho_{l}}{\rho_{v}-\rho_{l}}$ with Eq. (21) we get the cavitation erosion potential $\dot{e}_{p o t, d i v \bar{u}}$

$$
\dot{e}_{p o t, d i v \vec{u}}=\left(p_{d}-p_{v}\right) \frac{\rho}{\rho_{l}-\rho_{v}} \vec{\nabla} \cdot \vec{u} .
$$

Eq. (23) presents the second form of cavitation erosion potential, which is dependent on velocity divergence.

\subsubsection{Cavitation Erosion Potential $\dot{e}_{p o t, S_{\alpha v}}$}

Combining the conservation Eqs. (5) and (8) we get

$$
\frac{\partial \alpha}{\partial t}+\vec{u} \cdot \vec{\nabla} \alpha=\frac{\rho}{\rho_{l}} S_{\alpha v},
$$

and a third form of equation for erosion potential

$$
\dot{e}_{p o t, S_{\alpha v}}=\left(p_{d}-p_{v}\right) \frac{\rho}{\rho_{l}} S_{\alpha v} .
$$

In Eq. (25) the volume change is now expressed via the mass transfer source term $\mathrm{S}_{\alpha_{v}}$.

\subsection{Collapsing Bubble Energy Balance}

If it is assumed that, as the vapor cavity is collapsing, reduction of it's potential energy is converted instantaneously into the radiated power, then evaluation of Eqs. (22), (23) and (25) gives this instantaneous radiated power

$$
\dot{e}_{\text {rad }}=-\dot{e}_{p o t},
$$

where a negative sign is needed to obtain a positive value for instantaneous radiated power, as only a reduction in cavitation erosion potential $\dot{e}_{p o t}<0$ 
represents vapor cavity collapse, and only the collapse stage is considered in the model.

To predict the cavitation erosion on a solid surface better, the potential energy conservation hypothesis was introduced by Schenke et al. [25] and Mellisaris et al. [22]. It states that during the vapor cavity collapse potential energy is first converted into the kinetic energy of the vapor cavity interface, and is accumulating until the last moment of collapse, when it is finally released. This delayed release of energy from the collapsing cavity is named energy focusing, as the release of energy is more focused towards the center of the collapse compared to the case where energy is released instantaneously as described by Eq. (26). This accumulation and delayed release of energy is described by the additional conservation equation for collapse induced kinetic energy $\varepsilon$

$$
\frac{\partial \varepsilon}{\partial t}+\vec{\nabla} \cdot\left(\vec{u}_{i} \varepsilon\right)=-\dot{e}_{\text {rad }}(t)
$$

where $\vec{u}_{i}$ presents the collapse induced velocity, and $\dot{e}_{\text {rad }}(t)$ presents the radiated power of the pressure wave released in the bubble center at the final stage of collapse.

The discretized explicit model for transport Eq. (27) could be written as:

$$
\begin{aligned}
& \left.\varepsilon\right|_{t+\Delta t}=\left.\varepsilon\right|_{t}+\left.\frac{\partial \varepsilon}{\partial t}\right|_{t} \Delta t= \\
& =\left.\left(1-\left.\beta\right|_{t}\right)\left[(K-1) \dot{e}_{p o t} \Delta t-\varepsilon\left(P_{u}-1\right)\right]\right|_{t} .
\end{aligned}
$$

$P_{u}$ in Eq. (28) presents the projection operator, which ensures that the specific kinetic energy of the collapse accumulates at the inner side of the bubble interface, $K$ presents the conservation parameter that ensures global energy conservation and $\beta$ is the criterion which determines whether the induced energy is released at a given moment.

$$
\begin{aligned}
& P_{u}=\max \left[\frac{\vec{u} \cdot \vec{\nabla} \varepsilon}{|\vec{u}||\vec{\nabla} \varepsilon|}, 0\right], \\
& K=\frac{\int_{V} \frac{\varepsilon}{\Delta t} P_{u} d V}{\int_{V} \dot{e}_{p o t} d V}, \\
& \beta=\left\{\begin{array}{c}
1, \text { if } p>p_{\infty} \text { and } \alpha_{v}=0 \\
0, \text { else. }
\end{array}\right.
\end{aligned}
$$

$p_{\infty}$ in Eq. (29) presents the external pressure infinitely far from the bubble. This pressure represents the threshold for the propagation of the pressure wave outwards from the point of collapse, and the pressure at the exit from the domain was used for its value. The criterion $\alpha_{v}=0$ represents the end of the collapse when there is no more vapor phase in the computational cell.

The energy released after the collapse (at the end of time step $\Delta t$ ) is expressed as the specific power of the pressure wave

$$
\left.\dot{e}_{r a d}\right|_{t+\Delta t}=\frac{\left.(\beta \varepsilon)\right|_{t}}{\Delta t} .
$$

An infinite wave propagation speed is assumed in this model. The energy received by a surface element $S$ is then expressed in terms of the instantaneous surface specific impact power

$$
\dot{e}_{S}=\frac{1}{4 \pi} \int_{V} \dot{e}_{r a d}\left[\frac{\left(\vec{x}_{\text {cell }}-\vec{x}_{S}\right) \cdot \vec{n}}{\left|\vec{x}_{\text {cell }}-\vec{x}_{S}\right|^{3}}\right] d V,
$$

where $\vec{x}_{\text {cell }}$ is the position vector of the computational cell center, $\vec{x}_{S}$ is the position vector of the wall surface element center, and $\vec{n}$ is the surface element normal. By integrating Eq. (33) over the simulated time $(t)$, we obtain instantaneous surface specific impact energy with units $\left[\mathrm{J} / \mathrm{m}^{2}\right]$

$$
e_{S}=\int_{0}^{t} \dot{e}_{S} d t
$$

The described model to predict cavitation was implemented inside ANSYS Fluent via a UDF, which was written in the $\mathrm{C}$ programming language, compiled, and linked to the solver. Eqs. (22), (23), (25) and (28) to (34) were evaluated at the end of every time step. Energy accumulated on a given face of the surface is then stored as a sum of the previously stored value and newly computed value by Eq. (33).

\section{NUMERICAL TEST CASE AND SETUP}

\subsection{Geometry}

The conventional radial divergent test section was chosen for the test and validation of the cavitation erosion models [26]. The radial divergent test section geometry is shown in Fig. 1.

\subsection{Computational Grid and Boundary Conditions}

A computational grid of a $45^{\circ}$ sector was generated in ANSYS Fluent Meshing using ANSYS Mosaic meshing technology, which resulted in Poly-Hexcore grid topology. Additionally, a prismatic inflation layer was generated on the walls. The final mesh consisted of $1,396,703$ cells with a minimum orthogonal quality of 0.28 and maximum aspect ratio of 65 . The maximum wall $y^{+}$value was 51.9 and the minimum wall $y^{+}$value 
was 0.174 . Special care was taken to achieve $y^{+}<5$ around the radius before the radial divergent test section, and to achieve $30<y^{+}<300$ on the walls of the extended inlet and outlet parts of the computational domain. The computational domain was extended downstream with the outlet boundary positioned 100 $\mathrm{mm}$ in the radial direction from the radial divergent test section axis to improve numerical convergence. The mesh was refined around the radial divergent test section radius for improved resolution of cavitation dynamics, and, therefore, cavitation erosion prediction.

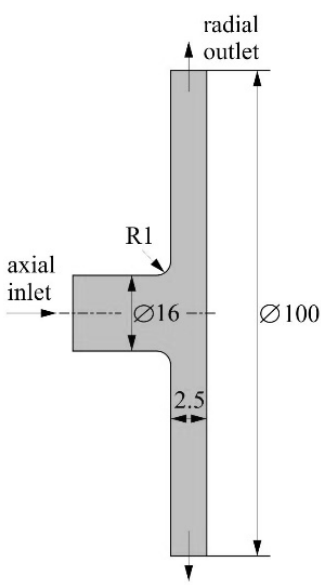

Fig. 1. Radial divergent test section

The results of the grid independence study are presented in Table 1. A medium grid was chosen as it provided us with an acceptable computation time and numerical uncertainty, as given by the grid convergence index (GCI). All simulations were performed on a personal computer using $10 \mathrm{CPU}$ cores of the Intel Core i9-10940X stock CPU with 64 GB of RAM. The time needed to obtain convergence on the medium mesh was approximately 10 days. For GCI calculations we chose the force exerted on the bottom plate, the fluid velocity at the outlet and turbulence kinetic energy at the outlet. In all three cases the estimated numerical error was less than $5 \%$ for both the medium and the fine grids [27].

Table 1. Computational grid independence study

\begin{tabular}{lcccc}
\hline Grid & $\begin{array}{c}\text { Number of } \\
\text { cells }\end{array}$ & $\begin{array}{c}\text { Force on } \\
\text { bottom } \\
\text { wall [N] }\end{array}$ & $\begin{array}{c}\text { Outlet } \\
\text { velocity } \\
{[\mathrm{m} / \mathrm{s}]}\end{array}$ & $\begin{array}{c}\text { Outlet turbulence } \\
\text { kinetic energy } \\
{[\mathrm{J} / \mathrm{kg}]}\end{array}$ \\
\hline Coarse & 475271 & 3802 & 3.64 & 0.196 \\
\hline Medium & 1396703 & 3798 & 3.57 & 0.195 \\
\hline Fine & 5175737 & 3799 & 3.51 & 0.187 \\
\hline GCImedium [\%] & - & 0.02 & 4.42 & 0.51 \\
\hline GClfine [\%] & - & 0.001 & 2.59 & 1.99 \\
\hline
\end{tabular}

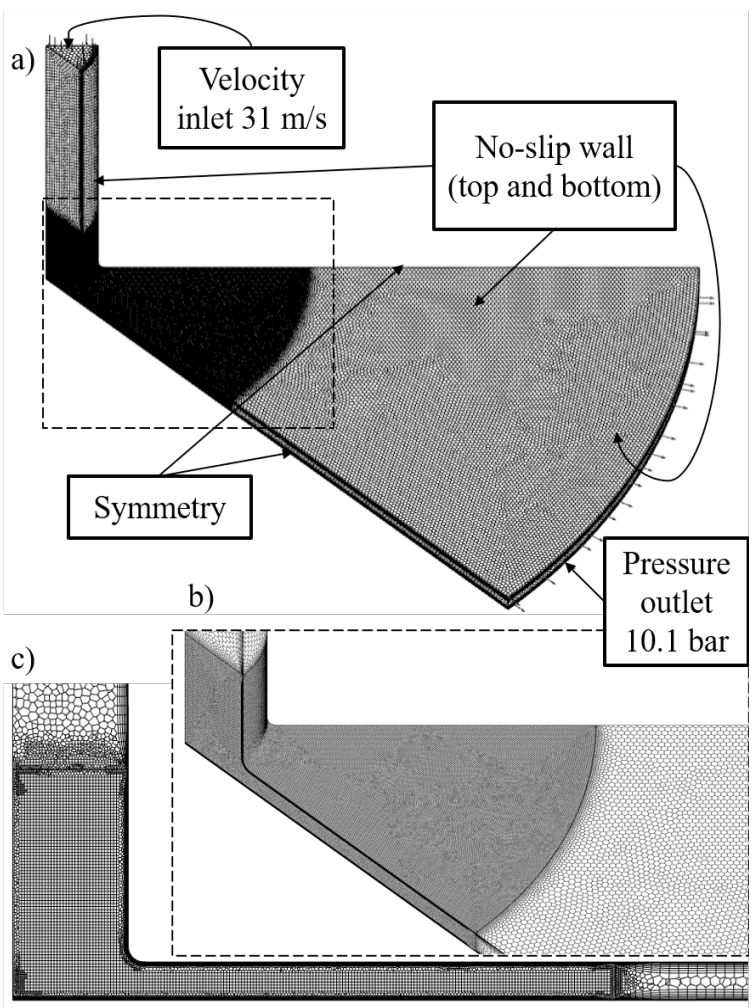

Fig. 2. Computational grid; a) isometric view of the whole grid with shown boundary conditions, b) detailed isometric view of the refined grid, and c) detailed front view of the refined grid

\subsection{Numerical Setup}

Solution of the Navier-Stokes equations was achieved using the SIMPLE algorithm, gradients were evaluated using the least squares cell-based method, interpolation of pressure cell face values was achieved by the PRESTO! interpolation scheme and the QUICK spatial discretization scheme was adopted for all equations. Temporal discretization of equations was achieved by the Bounded Second-Order Implicit time integration scheme.

A time step of $1 \cdot 10^{-6} \mathrm{~s}$ was chosen, which resulted in the maximum Courant number $C=1.59$. The iterative solution of equations within each time step was limited to 100 iterations, however, a scaled residuals convergence criterion of $1 \cdot 10^{-6}$ was achieved before this limitation. Overall, $0.012249 \mathrm{~s}$ of physical time were simulated.

\section{RESULTS}

The extent of cavitation predicted by the SchnerrSauer cavitation transport model at different times of numerical simulation is shown in Fig. 3. The 
black dotted line shows the cavitation closure line positioned $32 \mathrm{~mm}$ in a radial direction from the radial divergent test section axis, which was determined experimentally [26]. From the cavitation extent comparison, it is visible that the predicted cavitation sheet closure coincides with the experimental position, as cavitation number $\sigma=0.9$ was achieved. According to this, it can be concluded that the used cavitation model, boundary conditions and physical properties were determined properly to predict the cavitation dynamics correctly. Qualitative assessment of cavitation erosion by the numerical model is presented in Fig. 4, with three approaches for calculating cavitation erosion potential compared to the picture of the sample from the experiment by [26]. Both energy focusing, where radiated power was obtained from Eq. (32), and non-focusing approaches, where radiated power was obtained from Eq. (26), are

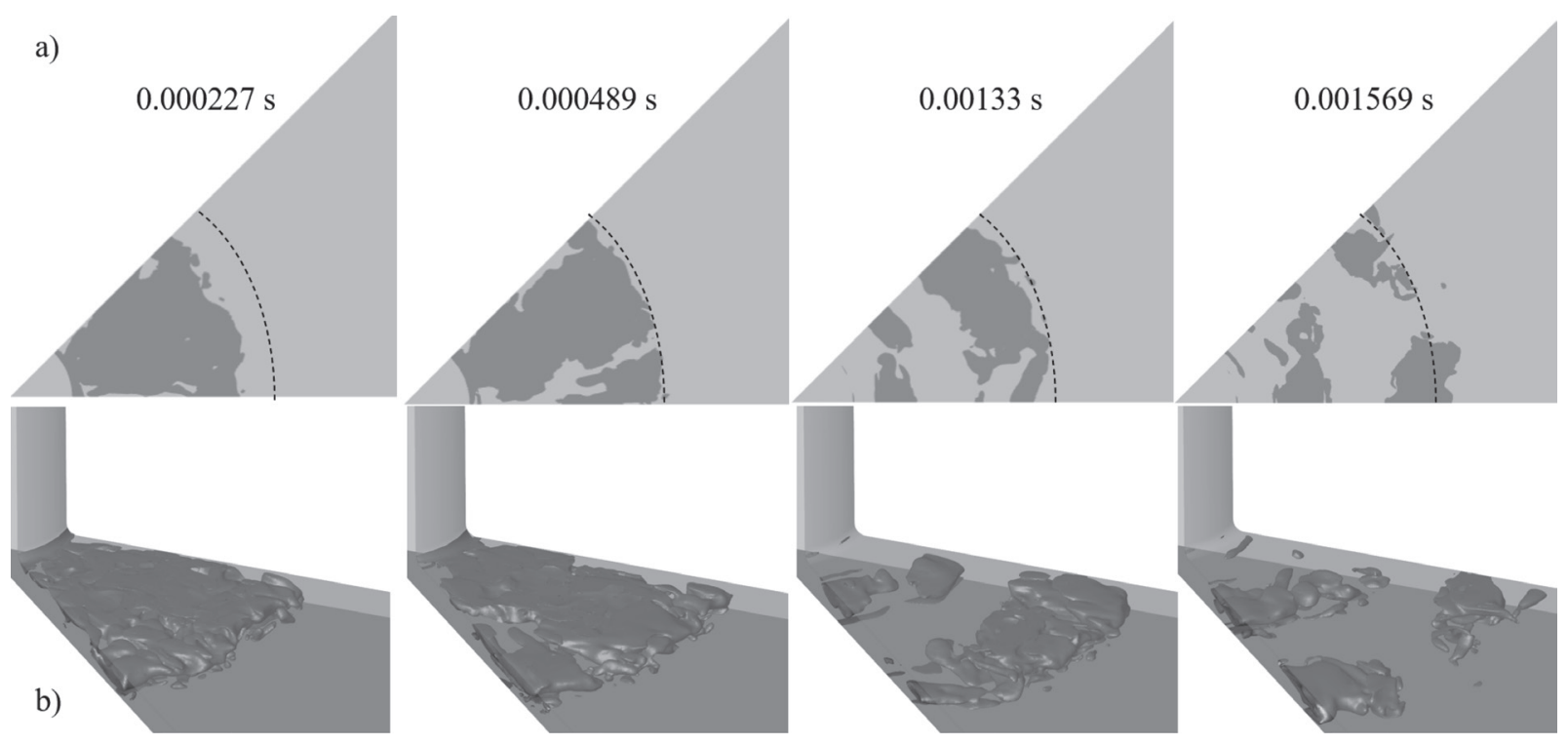

Fig. 3. Transient sheet cavitation displayed as an iso-surface of $\alpha=0.9$ at different times; a) top view; b) three- dimensional display of cavitation structures viewed from the side

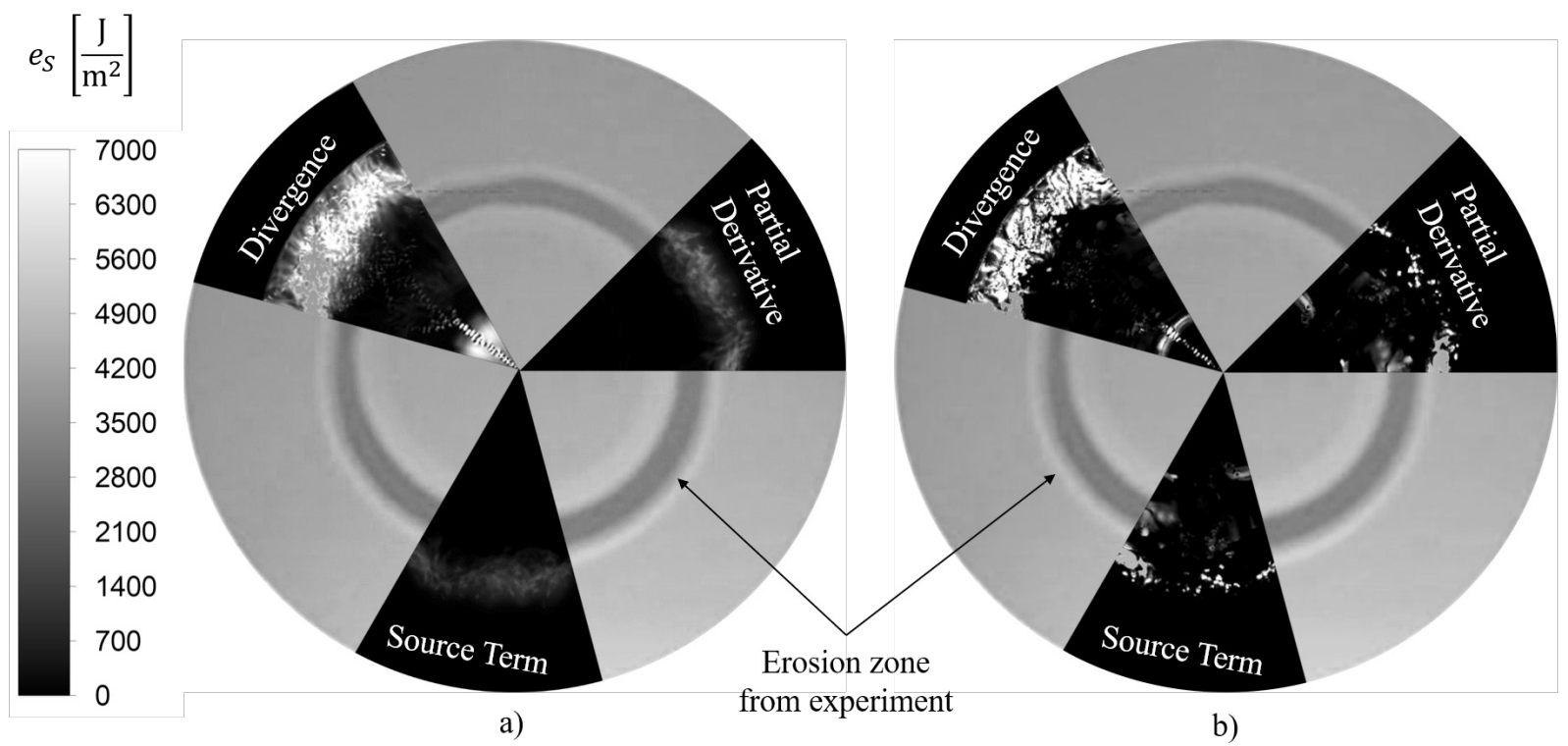

Fig. 4. Comparison between the cavitation erosion models and experiment [26]; a) accumulated energy on the surface without energy focusing; b) accumulated energy on the surface with energy focusing 
presented. Energy focusing resulted in a sharper, more pitted image compared to a more blended picture without energy focusing. Since in the case of energy focusing all previously accumulated collapse induced kinetic energy is released only at the final stage of collapse, it is reasonable to expect accumulation of radiated energy on the surface in a smaller area, and higher magnitude compared to the non-focusing case. In both cases, with energy focusing and without energy focusing, it is apparent that modelling cavitation erosion potential based on partial derivative of vapor volume fraction $(\partial \alpha / \partial t)$ and source term in vapor transport equation $\left(S_{\alpha v}\right)$ produced a similar erosion pattern and location of accumulated energy on the bottom plate surface, which are comparable with the experiment (shown in the background of Fig. 4). However, calculation based on the divergence of velocity $(\vec{\nabla} \cdot \vec{u})$ did not predict a similar erosion pattern and location of accumulation of energy on the bottom plate.

The comparison of the eroded zone position was similar for both (focusing and non-focusing) cases. The deviation of the position of the damaged area between the results of the numerical simulation and the experiment visible in Fig. 4 indicates a strong sensitivity of the numerical erosion prediction model on correct prediction of the cavitation dynamics.

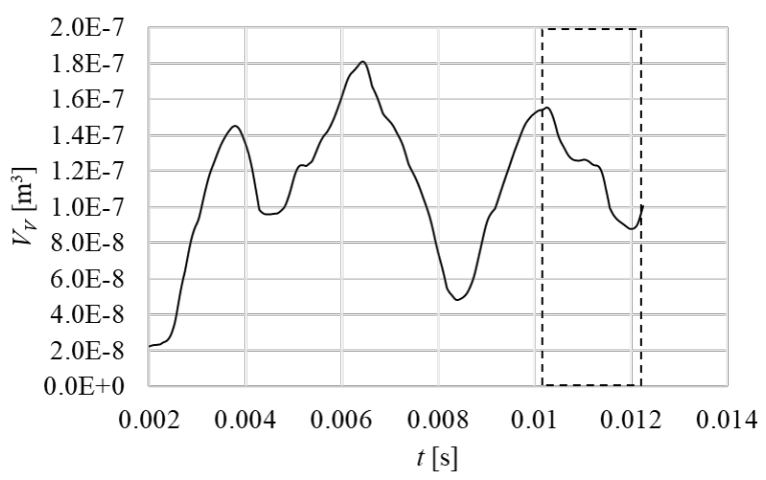

Fig. 5. Total vapor volume time evolution in the domain

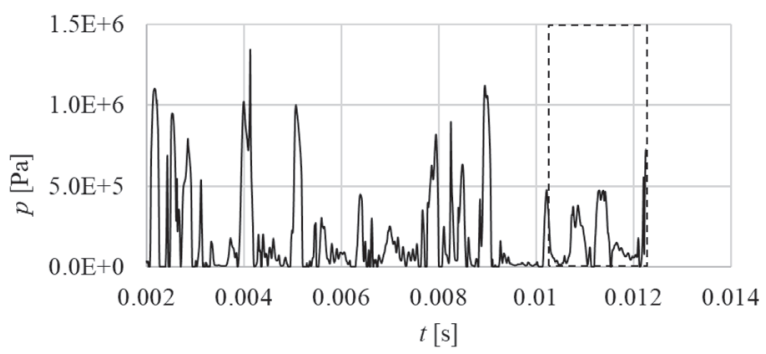

Fig. 6. Pressure time evolution, $20 \mathrm{~mm}$ from the radial divergent test section axis in a radial direction
The total vapor volume time evolution in the numerical domain is shown in Fig. 5 for the time interval $0.002 \mathrm{~s}$ to $0.012 \mathrm{~s}$. It is apparent that the total volume of vapor in the domain changes periodically, which confirms the transient nature of the extent of cavitation in the radial divergent test section. Additionally, Fig. 6 displays the local transient behavior of the vapor cloud shedding, with minimums in pressure between the peaks, indicating the presence of cavitation. It was observed that vapor cloud shedding occurs with higher frequency than the global (total) change in vapor volume in the domain. In a half of a total vapor volume change period (the dashed line frame in Figs. 5 and 6), at least three transitions occur between the liquid and vapor phases. Within the simulated time of $0.012249 \mathrm{~s}$ multiple vapor cloud shedding events occurred, which allowed for a consistent analysis of cavitation erosion.

Fig 7. shows the time evolution of the total cavitation potential power in the domain within this time period, calculated with a formulation based on the partial derivative of vapor volume fraction .

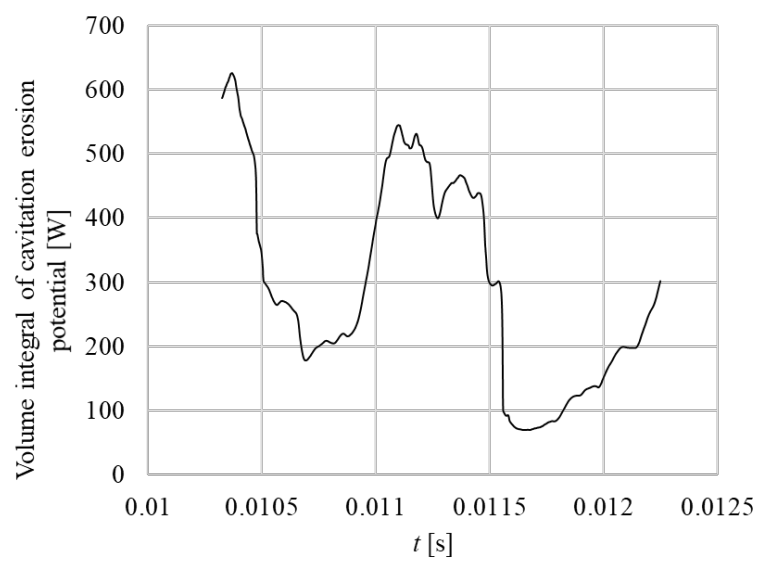

Fig. 7. Total cavitation potential power in the domain

Within the observed time window (the dash lined frame in Figs. 5 and 6) release of accumulated kinetic energy for the energy focusing case is presented in Fig. 8. Comparison between Figs. 7 and 8a further demonstrates and explains the difference in extent and magnitude of the predicted accumulation of energy between the energy focusing case and the nonfocusing case. In the non-focusing case cavitation potential power was released instantaneously, so Fig. 7. also represents the total radiated power of pressure waves in the domain, for the non-focusing case. It is again apparent that energy focusing results in fewer events with higher magnitude of pressure wave power radiation (at the end of vapor collapse). Because of 

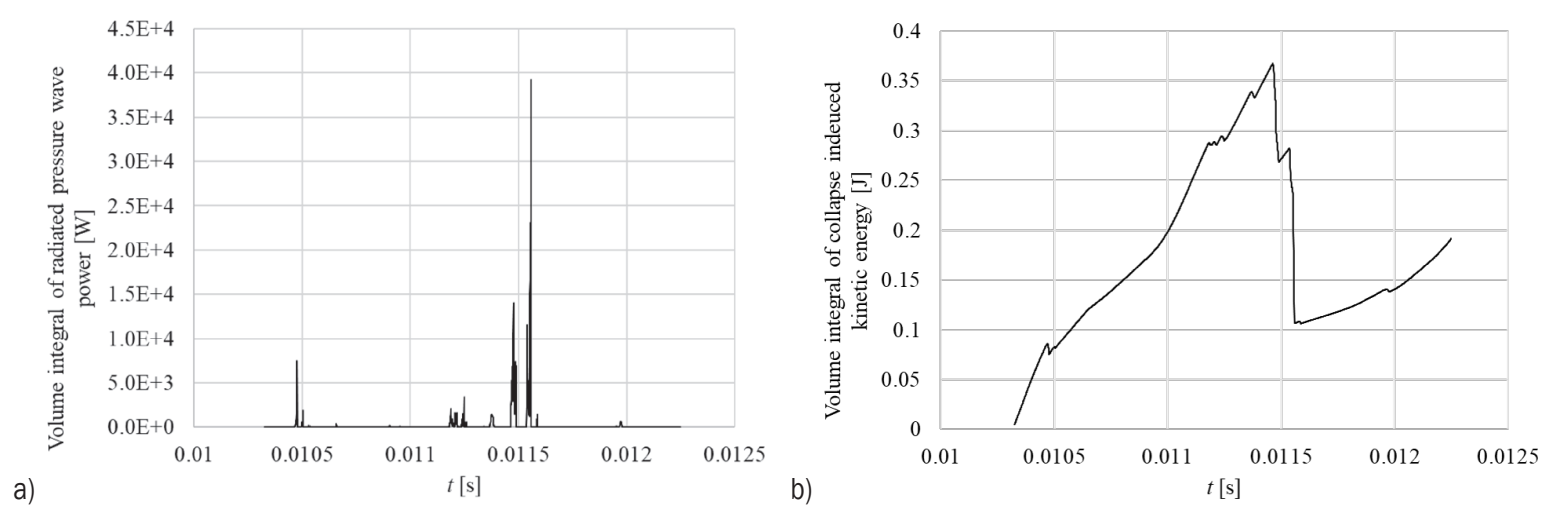

Fig. 8. a) Total radiated power of pressure waves generated by cavitation implosions in the domain; b) total vapor collapse induced kinetic energy of vapor in the domain

the energy conservation by the model, kinetic energy reduces at the end of vapor collapse when wave energy is released. Two pronounced events of release of energy are visible in Fig. 8. at around $0.0115 \mathrm{~s}$, first a smaller peak occurred, followed by a larger peak (Fig. $8 \mathrm{a})$. Hence, there are two pronounced corresponding drops in kinetic energy (Fig. 8b).

\section{DISCUSSION}

The presented study results show that the used mathematical-physical model with different formulations for prediction of vapor phase potential power, and, thus, cavitation erosion, predicts the position of damaged surfaces on the geometry of the radial divergent test section satisfactorily, which confirms the findings of other researchers on different flow cases [22] and [25] and different cavitation erosion models [16]. A more detailed comparison of the positions of damaged surfaces indicates discrepancies between the individual formulations. Given the fact that a verified cavitation transfer model was used, it can be concluded that the largest deviation of the damage positions from the experimentally observed ones occurred in the case of using a formulation with velocity divergence, both in the case of focusing and without focusing. The phenomenon was a consequence of numerical error, and is consistent with the observations of researchers who analyzed the flow over an isolated wing and marine propeller [22] and [25]. The formulations with the source term and the derivative of the vapor phase fraction confirm the importance of a correct description of the dynamics of cavitation structures. There was practically no difference in the calculated positions of damage between the applied formulations with energy focusing and without energy focusing. Conserving the potential energy of cavitation by focusing indicates an appropriate step towards a better agreement of the shape of the damaged surface with the experimental observations, where the damage was more concentrated and the eroded surface was characteristically pitted, while, in the case without focusing, the damage was more continuous and did not reflect the experimentally observed damage of eroded surfaces [26]. It has to be noted however, that to directly link the material pitting prediction to the cavitating flow simulation a change of scales would be necessary. Furthermore, material work hardening would have to be taken into account via proposed material strengthening models [26] and [28].

\section{CONCLUSIONS}

The performed analysis confirms that the formulation of cavitation erosion potential with the source term is comparable to the formulation with the derivative of vapor phase volume fraction in the case under consideration. An appropriate description of cavitation dynamics is crucial. The Schnerr-Sauer cavitation model predicts the extent and dynamics of cavitation correctly. In relation to that, it would be sensible to use cavitation transfer models of other authors, such as Singhal et al. [29] and Kunz et al. [30]. However, from the thermodynamic point of view this approach to model cavitation is not as appropriate, as discussed in [31]. The applied model transfers potential energy from cavitation structures to the surface, is energy conservative, which makes it a good basis for predicting the response of various materials exposed to the phenomenon of cavitation. In modelling their response to cavitation, the model used in this 
work could be upgraded by taking into account the mechanical properties of the material, which would allow the calculation of the actual depth of damage on different surfaces. This would allow a quantitative evaluation of the model and it's different formulations used in this work.

\section{ACKNOWLEDGEMENTS}

The authors wish to thank the Slovenian Research Agency (ARRS) for the financial support in the framework of the Research Programme P2-0196 Research in Power, Process and Environmental Engineering.

\section{REFERENCES}

[1] Lord Rayleigh, O.M.F.R.S. (1917). VIII. On the pressure developed in a liquid during the collapse of a spherical cavity. The London, Edinburgh, and Dublin Philosophical Magazine and Journal of Science, vol. 34, no. 200, p. 94-98, DOI:10.1080/14786440808635681.

[2] Plesset, M.S. (1949). The dynamics of cavitation bubbles. Journal of Applied Mechanics, vol. 16, no. 3, p. 277-282, DOl:10.1115/1.4009975.

[3] Hammitt, F.G. (1963). Observations on cavitation damage in a flowing system. Journal of Basic Engineering, vol. 85, no. 3, p. 347-356, Dol:10.1115/1.3656601.

[4] Vogel, A., Lauterborn, W., Timm, R. (1989). Optical and acoustic investigations of the dynamics of laser-produced cavitation bubbles near a solid boundary. Journal of Fluid Mechanics, vol. 206, p. 299-338, Dol:10.1017/S0022112089002314.

[5] Harrison, M. (1952). An experimental study of single bubble cavitation noise. The Journal of the Acoustical Society of America, vol. 24, p. 454-454, D0l:10.1121/1.1917513.

[6] Kornfeld, M., Suvorov, L. (1944). On the destructive action of cavitation. Journal of Applied Physics, vol. 15, no. 6, art. ID 495, DOI:10.1063/1.1707461.

[7] Plesset, M.S., Chapman, R.B. (1971). Collapse of an initially spherical vapour cavity in the neighbourhood of a solid boundary. Journal of Fluid Mechanics, vol. 47, no. 2, p. 283290, DOI:10.1017/S0022112071001058.

[8] Fortes Patella, R., Reboud, J.-L., Briancon-Marjollet, L. (2004). A Phenomenological and numerical model for scaling the flow agressiveness in cavitation erosion. Numerical Prediction of Cavitation Erosion, Delft.

[9] Leclercq, C., Archer, A., Fortes-Patella, R., Cerru, F. (2017). Numerical cavitation intensity on a hydrofoil for 3D homogeneous unsteady viscous flows. International Journal of Fluid Machinery and Systems, vol. 10, no. 3, p. 254-263, DOI:10.5293/IJFMS.2017.10.3.254.

[10] Carrat, J.-B., Fortes-Patella, R., Franc, J.-P. (2019). Experimental and numerical investigation of the erosive potential of a leading edge cavity. International Journal of Fluid Machinery and Systems, vol. 12, no. 2, p. 136-146, DOI:10.5293/IJFMS.2019.12.2.136.
[11] Schenke, S. van Terwisga, T.J.C. (2019). An energy conservative method to predict the erosive aggressiveness of collapsing cavitating structures and cavitating flows from numerical simulations. International Journal of Multiphase Flow, vol. 111, p. 200-218, D0l:10.1016/j.jjmultiphaseflow.2018.11.016.

[12] Li, Z. (2012). Assessment of Cavitation Erosion with a Multiphase Reynolds-averaged Navier-Stokes Method, PhD Thesis, TU Delft, Delft.

[13] Dular, M., Stoffel, B., Širok, B. (2006). Development of a cavitation erosion model. Wear, vol. 261, no. 5-6, p. 642-655, D0l:10.1016/j.wear.2006.01.020.

[14] Dular, M. Petkovšek, M. (2015). On the mechanisms of cavitation erosion - Coupling high speed videos to damage patterns. Experimental Thermal and Fluid Science, vol. 68, p. 359-370, DOI:10.1016/j.expthermflusci.2015.06.001.

[15] Jian, W., Petkovšek, M., Houlin, L., Širok, B., Dular, M. (2015). Combined numerical and experimental investigation of the cavitation erosion process. Journal of Fluids Engineering, vol. 137, no. 5, art. ID 051302, D0l:10.1115/1.4029533.

[16] Peters, A. el Moctar, 0. (2020). Numerical assessment of cavitation-induced erosion using a multi-scale Euler-Lagrange method. Journal of Fluid Mechanics, vol. 894, Dol:10.1017/ jfm.2020.273.

[17] Periasamy, G., Mouleeswaran, S., Venugopal, P., Perumal, C. (2021). Investigation of hydrodynamic flow characteristics in helical coils with ovality and wrinkles. Strojniški vestnik - Journal of Mechanical Engineering, vol. 67, no. 11, p. 570579, DOl:10.5545/sv-jme.2021.7374.

[18] Dai, Y., Zhang, Y.Y., Bian, J.N., Han, K., Zhu, X., Huang, Z.H., Xie, Y. (2021). CFD Simulation on hydrodynamics of underwater vehicle with ducted propellers. International Journal of Simulation Modelling, vol. 20, no. 3, p. 595-605, DOI:10.2507/IJSIMM20-3-C014.

[19] Brunhart, M., Soteriou, C., Daveau, C., Gavaises, M., Koukouvinis, P., Winterbourn, M. (2020). Cavitation erosion risk indicators for a thin gap within a diesel fuel pump. Wear, vol. 442-443, art. ID 20324, D0l:10.1016/j. wear.2019.203024.

[20] Gomez Santos, E., Shi, J., Venkatasubramanian, R., Hoffmann, G., Gavaises, M., Bauer, W. (2021). Modelling and prediction of cavitation erosion in GDi injectors operated with E100 fuel. Fuel, vol. 289, art. ID 119923, D0l:10.1016/j. fuel.2020.119923.

[21] Arabnejad, M.H., Svennberg, U., Bensow, R.E. (2021). Numerical assessment of cavitation erosion risk using incompressible simulation of cavitating flows. Wear, vol. 464465, art. ID 203529, DOI:10.1016/j.wear.2020.203529.

[22] Melissaris, T., Schenke, S., Bulten, N., van Terwisga, T.J.C. (2020). On the accuracy of predicting cavitation impact loads on marine propellers. Wear, vol. 456-457, art. ID 203393, D0l:10.1016/j.wear.2020.203393.

[23] Reboud, J.-L., Coutier-Delgosha, O., Benoit Stutz, B. (1998). Two-phase flow structure of cavitation: Experiment and modeling of unsteady effects. Third International Symposium on Cavitation, p. 1-7.

[24] Coutier-Delgosha, O., Fortes-Patella, R., Reboud, J.L. (2003). Evaluation of the turbulence model influence on the numerical simulations of unsteady cavitation. 
Journal of Fluids Engineering, vol. 125, no. 1, p. 38-45, DOl:10.1115/1.1524584.

[25] Schenke, S., Melissaris, T., van Terwisga, T.J.C. (2019). On the relevance of kinematics for cavitation implosion loads. Physics of Fluids, vol. 31, no. 5,, art. ID 052102, DOI:10.1063/1.5092711.

[26] Franc, J.-P. (2009). Incubation time and cavitation erosion rate of work-hardening materials. Journal of Fluids Engineering, vol. 131, no. 2, art. ID 021303, D0I:10.1115/1.3063646.

[27] Roache, P.J. (1994). Perspective: A method for uniform reporting of grid refinement studies. Journal of Fluids Engineering, vol. 116, no. 3, p. 405-413, DOl:10.1115/1.2910291.

[28] Liu, L., Guo, H., Yu, P. (2021). A model for material strengthening under the combined effect of cavitation-bubble collapse and $\mathrm{Al}_{2} \mathrm{O}_{3}$ particles, and its test verification. Strojniški vestnik - Journal of Mechanical Engineering, vol. 67, no. 1-2, p. 36-44, DOI:10.5545/sv-jme.2020.6937.

[29] Singhal, A.K., Athavale, M.M., Li, H., Jiang, Y. (2002) Mathematical basis and validation of the full cavitation model. Journal of Fluids Engineering, vol. 124, no. 3, p. 617-624, DOI:10.1115/1.1486223.

[30] Kunz, R.F., Boger, D.A., Stinebring, D.R., Chyczewski, T.S., Lindau, J.W., Gibeling, H.J., Venkateswaranm S., Govindan, T.R. (2000). A preconditioned Navier-Stokes method for two-phase flows with application to cavitation prediction. Computers \& Fluids, vol. 29, no. 8, p. 849-875, D0l:10.1016/ S0045-7930(99)00039-0.

[31] Goncalvès, E. Patella, R.F. (2011). Constraints on equation of state for cavitating flows with thermodynamic effects. Applied Mathematics and Computation, vol. 217, no. 11, p. 50955102, D0I:10.1016/j.amc.2010.07.056. 